\title{
ON THE THEOREM OF TUMURA-CLUNIE
}

\author{
BY HONG-XUN YI
}

\section{Introduction and Main Results.}

Let $f$ be a nonconstant meromorphic function in the complex plane. It is assumed that the reader is familiar with the notations of Nevanlinna theory (see, for example [3]). We denote by $S(r, f)$ any function satisfying $S(r, f)=$ $o(T(r, f))$ as $r \rightarrow+\infty$, possibly outside a set $E$ of finite linear measure. Throughout this paper we denote by $a_{j}(z)$ meromorphic functions which satisfying $T\left(r, a_{j}\right)=S(r, f)(j=0,1, \cdots, n)$. If $a_{n} \neq \equiv$, we call

$$
P[f]=a_{n} f^{n}+a_{n-1} f^{n-1}+\cdots+a_{1} f+a_{0}
$$

a polynomial in $f$ with degree $n$. If $n_{0}, n_{1}, \cdots, n_{k}$ are nonnegative integers, we call

$$
M[f]=f^{n_{0}}\left(f^{\prime}\right)^{n_{1}} \cdots\left(f^{(k)}\right)^{n_{k}}
$$

a differential monomial in $f$ of degree $\gamma_{M}=n_{0}+n_{1}+\cdots+n_{k}$ and of weight $\Gamma_{M}$ $=n_{0}+2 n_{1}+\cdots+(k+1) n_{k}$. If $M_{1}, \cdots, M_{n}$ are differential monomials in $f$, we call

$$
Q[f]=\sum_{j=1}^{n} a_{j}(z) M_{j}[f]
$$

a differential polynomial in $f$, and define the degree $\gamma_{Q}$ and the weight $\Gamma_{Q}$ by $\gamma_{Q}=\max _{j=1}^{n} \gamma_{M}$, and $\Gamma_{Q}=\max _{j=1}^{n} \Gamma_{M}$, respectively. If $Q$ is a differential polynomial, then $Q^{\prime}$ denotes the differential polynomial which satisfies $Q^{\prime}[f(z)]=\frac{d}{d z} Q[f(z)]$ for any meromorphic function $f$. (See, for example, Mues and Steinmetz [4, P 115]).

The following theorem was first stated by Tumura [6] and proved completely by Clunie [1]:

THEOREM A. Let $f$ and $g$ be entire functions, and

$$
F=a_{n} f^{n}+a_{n-1} f^{n-1}+\cdots+a_{1} f+a_{0} \quad\left(a_{n} \neq 0\right) .
$$

If $F=b e^{g}$, where $b(z)$ is a meromorphic function satisfying $T(r, b)=S(r, f)$, then

Received January 7, 1988, Revised July 12, 1988 


$$
F=a_{n}\left(f+\frac{a_{n-1}}{n a_{n}}\right)^{n} .
$$

Hayman proved the following theorem:

Theorem B (see [3, P 69-70]). Suppose that $f$ is meromorphic and not constant in the plane, that

$$
F=a_{n} f^{n}+a_{n-1} f^{n-1}+Q[f]
$$

where $Q[f]$ is a differential polynomial of degree at most $n-2$ in $f$. If $N(r, f)$ $+N\left(r, \frac{1}{F}\right)=S(r, f)$, then

$$
F=a_{n}\left(f+\frac{a_{n-1}}{n a_{n}}\right)^{n} .
$$

Mues and Steinmetz have given the following theorem:

THEOREM C (see [4]). Let $f$ be a nonconstant meromorphic function. Suppose that $F$ is given by (3). If $\bar{N}(r, f)+\bar{N}\left(r, \frac{1}{F}\right)=S(r, f)$, then

$$
F=a_{n}\left(f+\frac{a_{n-1}}{n a_{n}}\right)^{n} \text {. }
$$

Toda proved the following theorem:

THEOREM D (see [5]). Let $f$ be a nonconstant meromorphic function. Suppose that $F$ is given by (3). If

then

$$
\limsup _{\substack{r \rightarrow \infty \\ r \notin E}} \frac{\bar{N}\left(r, \frac{1}{F}\right)+2 \bar{N}(r, f)}{T(r, f)}<\frac{1}{2},
$$

$$
F=a_{n}\left(f+\frac{a_{n-1}}{n a_{n}}\right)^{n} .
$$

Recently Weissenborn has given the following theorem:

THEOREM E (see [7]). Let $f$ be a nonconstant meromorphic function. Suppose that $F$ is given by (3). Then either

$$
T(r, f) \leqq \bar{N}\left(r, \frac{1}{F}\right)+\bar{N}(r, f)+S(r, f),
$$

or

$$
F=a_{n}\left(f+\frac{a_{n-1}}{n a_{n}}\right)^{n}
$$


In this paper we improve the above results and obtain the following:

THEOREM. Suppose that $f$ is a nonconstant meromorphic function, that $F$ is given by (4). If

$$
\limsup _{\substack{r \rightarrow \infty \\ r \notin E}} \frac{\bar{N}\left(r, \frac{1}{F}\right)+\alpha \bar{N}(r, f)+\bar{N}\left(r,\left(f+\frac{a_{n-1}}{n a_{n}}\right)^{-1}\right)}{T(r, f)}<2,
$$

where $\alpha=\max \left\{1, \Gamma_{Q}+3-n\right\}, \Gamma_{Q}$ is the weight of $Q[f]$, then

$$
F=a_{n}\left(f+\frac{a_{n-1}}{n a_{n}}\right)^{n} .
$$

The proof of the Theorem is left to $\S 4$. In the special case that $F$ is given by (3), our result is

COROLlary. Suppose that $f$ is a nonconstant meromorphic function and that $F$ is given by (3). If

then

$$
\limsup _{\substack{r \rightarrow \infty \\ r \notin E}} \frac{\bar{N}\left(r, \frac{1}{F}\right)+\bar{N}(r, f)+\bar{N}\left(r,\left(f+\frac{a_{n-1}}{n a_{n}}\right)^{-1}\right)}{T(r, f)}<2
$$

$$
F=a_{n}\left(f+\frac{a_{n-1}}{n a_{n}}\right)^{n} .
$$

The above Corollary improves Theorems A, C, D and E. To illustrate our results we give an example.

Let $f(z)=e^{z}, F=f^{n}+f^{n-2}(n \geqq 2)$, we can easily verify

$$
\limsup _{\substack{r \rightarrow \infty \\ r \notin E}} \frac{\bar{N}\left(r,-{ }^{-}\right)+\alpha \bar{N}(r, f)+\bar{N}\left(r,\left(f+\frac{a_{n-1}}{n a_{n}}\right)^{-1}\right)}{T(r, f)}=2 .
$$

This example shows that our results are sharp.

\section{Some Lemmas.}

The following four lemmas will be needed in the proof of our Theorem.

LEMMA 1 (see [2]). Let $f$ be a meromorphic function, and $Q[f]$ be a differential polynomal in $f$ of degree $\gamma_{Q}$. Then

$$
m(r, Q[f]) \leqq \gamma_{Q} m(r, f)+S(r, f) .
$$

LeMma 2. Suppose that $M[f]$ is given by (1). If $f$ has a pole at $z=z_{0}$ of order $p$, then $z_{0}$ is a pole of $M[f]$ of order $(p-1) \gamma_{M}+\Gamma_{M}$. 
Proof. Obviously, the order of $M[f]$ at the pole $z_{0}$ is

$$
p n_{0}+(p+1) n_{1}+\cdots+(p+k) n_{k}=(p-1) \gamma_{M}+\Gamma_{M} .
$$

LEMMA 3. Suppose that $Q[f]$ is given by (2). Let $z_{0}$ be a pole of $f$ of order $p$, and not a zero nor a pole of coefficients of $Q[f]$. Then $z_{0}$ is a pole of $Q[f]$ of order at most $p \gamma_{Q}+\left(\Gamma_{Q}-\gamma_{Q}\right)$.

Proof. By Lemma 2, $z_{0}$ is a pole of $M_{j}[f]$ of order $(p-1) \gamma_{M_{j}}+\Gamma_{M_{j}}(j=$ $1,2, \cdots, n)$. Therefore, $z_{0}$ is a pole of $Q[f]$ of order at most

$$
\max \left\{(p-1) \gamma_{M},+\Gamma_{M_{j}}\right\} \leqq(p-1) \gamma_{Q}+\Gamma_{Q}=p \gamma_{Q}+\left(\Gamma_{Q}-\gamma_{Q}\right) \text {, }
$$

which proves Lemma 3.

LEMMA 4. Let $f$ be a nonconstant meromorphic function, and $F=f^{n}+Q[f]$, where $Q[f]$ is a differential polynomial in $f$ of degree $\gamma_{Q}$ and of weight $\Gamma_{Q}$. If $Q[f] \neq 0$, then

$$
\left(n-\gamma_{Q}\right) T(r, f) \leqq \bar{N}\left(r, \frac{1}{F}\right)+\bar{N}\left(r, \frac{1}{f}\right)+\left(\Gamma_{Q}-\gamma_{Q}+1\right) \bar{N}(r, f)+S(r, f) .
$$

The proof of Lemma 4 is given in $\S 3$.

\section{Proof of Lemma 4.}

If $n \leqq \gamma_{Q}$, the conclusion of Lemma 4 holds obviously. In the following we suppose $n>\gamma_{Q}$. By $F=f^{n}+Q[f]$, we have

$$
F^{\prime}=\frac{F^{\prime}}{F} f^{n}+\frac{F^{\prime}}{F} Q[f], \quad F^{\prime}=n f^{n-1} f^{\prime}+Q^{\prime}[f],
$$

and hence

$$
f^{n}\left(\frac{F^{\prime}}{F}-\frac{n f^{\prime}}{f}\right)=Q[f]\left(\frac{Q^{\prime}[f]}{Q[f]}-\frac{F^{\prime}}{F}\right) .
$$

Let

Then

$$
\Omega_{1}[f]=\frac{F^{\prime}}{F}-\frac{n f^{\prime}}{f}, \quad \Omega_{2}[f]=Q[f]\left(\frac{Q^{\prime}[f]}{Q[f]}-\frac{F^{\prime}}{F}\right) .
$$

$$
f^{n} \Omega_{1}[f]=\Omega_{2}[f] .
$$

If $\Omega_{1}[f] \equiv 0$, then $\Omega_{2}[f] \equiv 0$. By integration we get

and hence

$$
f^{n}=c Q[f] \quad(c \neq 0),
$$

that is

$$
T\left(r, f^{n}\right)=T(r, Q[f])+O(1)
$$

$$
n T(r, f)=m(r, Q[f])+N(r, Q[f])+O(1) .
$$


Using Lemma 1 and Lemma 3, we have

$$
\begin{aligned}
& m(r, Q[f]) \leqq \gamma_{Q} m(r, f)+S(r, f), \\
& N(r, Q[f]) \leqq \gamma_{Q} N(r, f)+\left(\Gamma_{Q}-\gamma_{Q}\right) \bar{N}(r, f)+S(r, f) .
\end{aligned}
$$

From the above we get

$$
\left(n-\gamma_{Q}\right) T(r, f) \leqq\left(\Gamma_{Q}-\gamma_{Q}\right) \bar{N}(r, f)+S(r, f),
$$

the conclusion of Lemma 4 holds. In the following we suppose that $\Omega_{1}[f] \neq 0$. Noting $\Omega_{1}[f]=\frac{F^{\prime}}{F}-\frac{n f^{\prime}}{f}$, we have $m\left(r, \Omega_{1}[f]\right)=S(r, f)$. From

we have

$$
\Omega_{2}[f]=Q[f]\left(\frac{Q^{\prime}[f]}{Q[f]}-\frac{F^{\prime}}{F}\right)
$$

$$
\begin{aligned}
m\left(r, \Omega_{2}[f]\right) & \leqq m(r, Q[f])+S(r, f) \\
& \leqq \gamma_{Q} m(r, f)+S(r, f),
\end{aligned}
$$

using Lemma 1. By (5) we have $f^{n}=\frac{\Omega_{2}[f]}{\Omega_{1}[f]}$, and hence

that is

$$
m\left(r, f^{n}\right) \leqq m\left(r, \Omega_{2}[f]\right)+m\left(r, \frac{1}{\Omega_{1}[f]}\right),
$$

$$
n m(r, f) \leqq \gamma_{Q} m(r, f)+m\left(r, \frac{1}{\Omega_{1}[f]}\right)+S(r, f) .
$$

Again by the first fundamental theorem (see [3]), we get

$$
m\left(r, \frac{1}{\Omega_{1}[f]}\right)=N\left(r, \Omega_{1}[f]\right)-N\left(r, \frac{1}{\Omega_{1}[f]}\right)+S(r, f) .
$$

Obviously, a pole of $\Omega_{1}[f]$ occurs at one of the zeros of $F$ and $f$, poles of $f$, zeros and poles of coefficients of $Q[f]$. Let $z_{o}$ be a pole of $f$ of order $p$, and not a zero nor a pole of coefficients of $Q[f]$. Then $z_{0}$ is a pole of $f^{n}$ of order $p n$. From Lemma 3 we know that $z_{0}$ is a pole of $\Omega_{2}[f]$ of order at most $p \gamma_{Q}+\left(\Gamma_{Q}-\gamma_{Q}\right)+1$. If $z_{0}$ is a pole of $\Omega_{1}[f]$, since $\Omega_{1}[f]=\frac{\Omega_{2}[f]}{f^{n}}, z_{0}$ is the pole of $\Omega_{1}[f]$ of order at most

$$
p \gamma_{Q}+\left(\Gamma_{Q}-\gamma_{Q}+1\right)-p n=\left(\Gamma_{Q}-\gamma_{Q}+1\right)-p\left(n-\gamma_{Q}\right) .
$$

If $z_{0}$ is not a pole of $\Omega_{1}[f]$, since

$$
\frac{1}{\Omega_{1}[f]}=\frac{f^{n}}{\Omega_{2}[f]}
$$

$z_{0}$ is a zero of $\Omega_{1}[f]$ of order at least 


$$
p n-\left\{p \gamma_{Q}+\left(\Gamma_{Q}-\gamma_{Q}\right)+1\right\}=p\left(n-\gamma_{Q}\right)-\left(\Gamma_{Q}-\gamma_{Q}+1\right) \text {. }
$$

Hence we have

$$
\begin{aligned}
N\left(r, \Omega_{1}[f]\right)-N\left(r, \frac{1}{\Omega_{1}[f]}\right) \leqq & \bar{N}\left(r, \frac{1}{F}\right)+\bar{N}\left(r, \frac{1}{f}\right) \\
& +\left(\Gamma_{Q}-\gamma_{Q}+1\right) \bar{N}(r, f)-\left(n-\gamma_{Q}\right) N(r, f)+S(r, f) .
\end{aligned}
$$

From the above we get

$$
\left(n-\gamma_{Q}\right) T(r, f) \leqq \bar{N}\left(r, \frac{1}{F}\right)+\bar{N}\left(r, \frac{1}{f}\right)+\left(\Gamma_{Q}-\gamma_{Q}+1\right) \bar{N}(r, f)+S(r, f) .
$$

This completes the proof of Lemma 4 .

\section{Proof of the Theorem.}

Let $g=f+\frac{a_{n-1}}{n a_{n}}$, and $G=\frac{F}{a_{n}}$; then

$$
G=g^{n}+Q^{*}[g] \text {, }
$$

where $Q^{*}[g]$ is a differential polynomial in $g$ of degree $\gamma_{Q^{*}}$ and of weight $\Gamma_{Q^{*} \text {. }}$ Obviously,

$$
\begin{aligned}
& \gamma_{Q^{*}} \leqq n-2 \\
& \Gamma_{Q^{*}} \leqq \max \left\{n-2, \Gamma_{Q}\right\} .
\end{aligned}
$$

If $\Gamma_{Q}>n-2$, then $\Gamma_{Q^{*}}=\Gamma_{Q}, \alpha=\Gamma_{Q}+3-n$. If $\Gamma_{Q} \leqq n-2$, then $\Gamma_{Q^{*}} \leqq n-2, \alpha=1$. Therefore, $\Gamma_{Q^{*}}-\gamma_{Q^{*}}+1 \leqq \alpha+\left(n-2-\gamma_{Q^{*}}\right)$.

Suppose $Q^{*}[g] \neq 0$. From Lemma 4 , we have

$$
\begin{aligned}
\left(n-\gamma_{Q^{*}}\right) T(r, g) \leqq & \bar{N}\left(r, \frac{1}{G}\right)+\bar{N}\left(r, \frac{1}{g}\right)+\left(\Gamma_{Q^{*}}-\gamma_{Q^{*}}+1\right) \bar{N}(r, g)+S(r, g) \\
\leqq & \bar{N}\left(r, \frac{1}{G}\right)+\bar{N}\left(r, \frac{1}{g}\right)+\alpha \bar{N}(r, g) \\
& +\left(n-2-\gamma_{Q^{*}}\right) \bar{N}(r, g)+S(r, g) \\
\leqq & \bar{N}\left(r, \frac{1}{G}\right)+\bar{N}\left(r, \frac{1}{g}\right)+\alpha \bar{N}(r, g) \\
& +\left(n-2-\gamma_{Q^{*}}\right) T(r, g)+S(r, g) .
\end{aligned}
$$

Thus, we have

$$
2 T(r, g) \leqq \bar{N}\left(r, \frac{1}{G}\right)+\bar{N}\left(r, \frac{1}{g}\right)+\alpha \bar{N}(r, g)+S(r, g) .
$$

Noting 


$$
\begin{aligned}
& T(r, g)=T(r, f)+S(r, f), \\
& \bar{N}\left(r, \frac{1}{G}\right)=\bar{N}\left(r, \frac{1}{F}\right)+S(r, f), \\
& \bar{N}\left(r, \frac{1}{g}\right)=\bar{N}\left(r,\left(f+\frac{a_{n-1}}{n a_{n}}\right)^{-1}\right), \\
& \bar{N}(r, g)=\bar{N}(r, f)+S(r, f),
\end{aligned}
$$

we get

$$
2 T(r, f) \leqq \bar{N}\left(r, \frac{1}{F}\right)+\alpha \bar{N}(r, f)+\bar{N}\left(r,\left(f+\frac{a_{n-1}}{n a_{n}}\right)^{-1}\right)+S(r, f) .
$$

So

$$
\limsup _{\substack{r \rightarrow \infty \\ r \notin E}} \frac{\bar{N}\left(r, \frac{1}{F}\right)+\alpha \bar{N}(r, f)+\bar{N}\left(r,\left(f+\frac{a_{n-1}}{n a_{n}}\right)^{-1}\right)}{T(r, f)} \geqq 2,
$$

which is a contradiction. This shows that $Q^{*}[g] \equiv 0$, that is

$$
F=a_{n}\left(f+\frac{a_{n-1}}{n a_{n}}\right)^{n} .
$$

The Theorem is thus proved.

\section{REFERENCES}

[1] J. Clunie, On integral and meromorphic functions, J. London Math. Soc., 37 (1962), 17-27.

[2] W. Doeringer, Exceptional values of differential polynomials, Pacific J. Math. 98 (1982), 55-62.

[3] W.K. Hayman, Meromorphic Functions, Oxford, Clarendon Press, 1964.

[4] E. Mues and N. Steinmetz, The theorem of Tumura-Clunie for meromorphic functions, J. London Math. Soc. 23 (1981), 113-122.

[5] N. TODA, On an extension of the Theorem of Tumura-Clunie, Contemporary Mathematics, 25 (1983), 215-219.

[6] Y. Tumura, On the extensions of Borel's theorem and Saxer-Csillag's theorem, Proc. Phys. Math. Soc. Japan 19 (1937), 29-35.

[7] G. Weissenborn, On the Theorem of Tumura and Clunie, Bull. London Math. Soc. 18 (1986), 371-373.

Department of Mathematics

SHANDONG UNIVERSITY

Jinan, Shandong, P.R. OF China 